\title{
THEORETICAL INTERPRETATION OF HIGH-ENERGY NUCLEAR COLLISIONS
}

\author{
Progreas Report
}

July 1,1891 - June 30,1982

George Fai

Kent State University

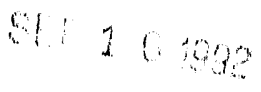

June 1992

Prepased for the U.S. Department of Energy

Grant No. DE-FG02-86ER40251

\section{DISCLAIMER}

This report was prepared as an account of work sponsored by an agency of the United States Government. Neithar the United States Government nor any agency thereof, nor any of their employees, mekes any warranty, express o": implied, or assumes any legal liability or responsibility for the accuracy, completeness, or usefulness of any information, apparatus, product, of process disciosed, or represents that its use would not infringe privalely owned rights. Reference herein to any specific commercial product, process, or service by trade nume, trademark. manufactures, or otherwise does not nocessarily constitute or imply its endorsement, recomsmendation, of favoring by the United States Government or alyy agency thereof. The views and opinions of authors expressed herein do not necessarily state or reflect those of the United States Government of any agency therwof. 


\begin{abstract}
Nuelear eollisions are interpreted theoretically. The nucear equation of atate is atudied in a wide energy range. Subnucleonic degrees of freedom are invaked at high energy densititu and at short leagth-reales. Questions of dyamical colliaion nimulations ase investigated. Direet sopport is provided for experiment in the form of collaborative projects. The major objective of this nuclear theory program is a better underatanding of the properties of otrongly interacting matter on the muclear energy ocale, es manifested in high-energy heary-ion collivions.
\end{abstract}

This progress report is a brief nummary of the renearch results and progress in the time period July 1, 1991 - June 30, 1992. Furthernore, in the second part of the report, I will outline sorne research topics of new and continuing interest to review ongoing and proposed projects for the next budget period.

The results of the research activitien of the principal investigator and his postdoctoral associate during the time period indicated have been partly published. Four further papers are accopted for publication. Several contributions to the proceedings of acientific meetings are also in press, in connection with the corresponding presentations at thene meetings. Other manuscripts have just been submitted for publication.

\title{
1 Results
}

The quark-gluon substructure of wuclear matter and nuclei is emerging an a central question of nuclear physics. This substructure can hopefully be revealed in the laboratory by studying energetic nuclear collisions. Accordingly, the principal investigator continues to be active in both (i) the theorexical modeling of submwcleonic degrees of treedom, and (ii) the phenomenology of nuclear collisions. Feaction mechanims, dynamical simulations and fragmentation need to be better understood at all energies to have a chance of success in identifying interesting phenomena. Advancer in the field are produced by the interaction between experiment and theory. Providing direct support for experinent therefore remains a high priority of this program. 
In the following, I list publicstions in the reporting period in the categories of journal articles, contributions, and abstracts. Next, I briefly comment on those ureas whera, in ray judgement, important new developments have occurred since the submission of my previous progress report (July 1991).

Refereed Journals:

1. 'Pion Yields and Pion Spectra from Nuclens Collisions' A.F. Barghouty, G. Fal, and D. Keane, Nucl. Phyn. As35, 715 (1991).

2. 'High-Order Collective Flow Correlation in Heavy-Ion Collisions,' J. Jiang, D. Beavis, S.V. Chu, G. Fai, S.Y. Fung, Y.Z. Yiang, D. Kease, Q.J. Liu, Y.M. Liu, Y. Sheo, M. Vient, and S. Wang, Phys. Rev. Lett. 68, 2739 (1992).

3. 'Nuclear Equation of Stmte with Mornentum-Dependent Inferactions,' L.P. Cremai, G. Fai, C. Gale, and E. Onnes, Phys. Rev. C in press, preprint KSUCNR-008-91 (December 1991).

4. 'Measuring the QCD Transition Temperature with Dileptons in UItra-Relativistic Nuclear Collisions,' D. Seibert, V.K. Mishra, and G. Fai, Phys. Rev. C in press, preprint KSUCNR-002-92 (February 1992).

5. 'Momentura-Dependent Interactions and the Nuclear Equation of State,' V.K. Mishra and G. Faj, Revista Mexicana de Fisica, in press, preprint KSUCNR-018-91 (December 1991).

6. 'Nonlocal Fieid-Theory Model for Nuclear Matter,' V.K. Mishrs, G. Fui, P.C. Taudy, and M.R. Frank, Phys. Rev. C accepted, preprint KSUCNR-006-02 (March 1992).

7. 'How to Measure the QCD Transition 'Temperature,' D. Scibert, Phys. Rev. Lett. 68 $1476(1992)$.

8. 'Robustness of Covariance Matrix Techniques,' D. Seibert, submitted for publication, preprint KSUCNR-014-91 (Novembex 1991).

9. 'High-Energy Phatons as a Thermorneter for Ultru-Relativistic Nuclear Collisions,' D. Seiber, submitted for publication, preprint KSUCNR-004-92 (February 1992). 
10. 'Measuring Velocity Ratios with Correlation Functions,' subroitted for publication, D. Seibert, K. Haglin and C. Gale, preprint KSUCNR-005-92 (March 1992).

Published Contributions:

11. 'Hadron-Quark Phase Transition with a Momentum-Dependent Yukawa Interaction for Hadrons,' L.P. Ciernaj, G. Fai, C. Gale, Gy. Kluge, V.K. Mishra, and E. Oszes, Proceedings of the Workehop on Relativintic Bleavy Ion Physics at Present and Future Accelerators, Budapest, Hungary, June 1891 (CRTP, 1991; T. Cøörgö, S. Hegyi, B. Lukács, and J. Zimányi, eds.), p. 124.

12. 'Studying Ultra-Relativistic Nuclear Collisions with Dileptons and Photons,' D. Seibert, Proceedings of the $8^{\text {th }}$ Winter Workshop on Nuclear Dyramics, Jackson Hole, Wyoming, January 1992 (World Scientific, 1992: W. Bauer and B. Back, eds.), in press, preprint KSUCNR-003-92 (February 1992).

13. 'Rapidity Correlations in Ultra-Relativistic Nuclear Collisions,' D. Seibert, Proceedings of Quark Matter '01, Gatlinburg, Tennessee, November 1991 (Nucl. Phys. A; T.C. Awes, F.E. Obenshain, F. Plasil, M.R. Strayer and C.Y. Wong, eds.), in press, preprint KSUCNR-01.2-91 (November 1991).

Abstracts:

14. 'A Thermometer for the QCD Trantition Temperature,' D. Seibert, V.K. Mishra, and G. Fai, Proceedingw of the 1092 International Nuclear Physics Conference, July 26 - August 1, 1992, Wiesbaden, Germany

15. 'Lagrangian Model of the Pauli Potential,' J.J. Neurnann and G. Fai, Bull. Am. Phy's. Soc. 37, 931 (1992).

16. 'Relativistic Hastree Approzimation in QHD with Sideways Form Factors,' V.K. Mishra, P.C. Tandy, and G. Fai, Bull. Am. Phys. Soc. \$7, 1040 (1992).

The first itern of the above list represents work carried out in the previous budget period and is discussed in my July "91 progress report. It is included here with the full bitliographic information for completeness. 


\section{Interpretation of data at BEVALAC energies}

The observation of nuclear matter flow (the collective sidewards deflection of the reaction products) is frequently hailed as the most important result of the experimental program at the BEVALAC. Nevertheless, previous studies of this effect have not ruled out the possibility that the flow effect is dominated by a small number of correlated fragrnents in each event, as opposed to being a collective motion to which most or ull frapunents contribute. In a recent Phys. Rev, Letter (item 2), we use $4 \pi$ datn from the BEVALAC strearner chamber to study wimuthal correlation for fragment paire and higher-order multiplets. Based on a imulation we infer that more than $70 \%$ of the forward-going fragments from collitions of Ar on $\mathrm{Pb}$ at $0.4 \mathrm{GeV} /$ nucleon carry a component of the collective motion. The simulation we utilize is the FREESCO code developed earlier by the principal investigator and J. Randrup of the Lawrence Berkeley Laboratory. The work reported on in item 2 was carried out in collaboration with experimentalists, in particulas with D. Keane, the other DoE funded faculty member at KSU. The crew included several graduate students, among them Y. Shao, who is coadvised by $D$. Keane and the principal investigator.

The flow of nuclear matter is intimately related to its equation of state (EOS). The incompressibility of nuclear matter is frequently taken to be the most important indicator of the static properties of nuclear matter. This EOS information is extracted from BEVALAC and other collision dat vio dynamical simulations using effective interactions as input. There is a qualitative change in the results when momentum dependence is allowed in the effective interaction. With an eye on interpreting BEVALAC data, we have utudied the equilibrium properties of momentum-dependent interactious. Progress in this ares will be deacribed next.

\section{Momentum-dependent interactions}

Momentum.dependent mean fields play an increasing role in the description of nuclear collisions at BEVALAC energies. The mominnturn dependence bas important implications on the EOS. In addition, in a momentum-dependent mean field, the momentum distribution of nucleons at finite temperature is more complicated than a simple Maxwellian. This may change production cross sections of recent intes'est. We have studied (itens 3,5 and 11 ) the equilibrium properties of nuclear matter with in momentum-dependent effective interaction, referred 
to as MDYI, in the Boltzmann approximation. We have shown that the zero-temperature optical potential nbtained from the MDYI describes data satisfactorily up to energies on the GeV/nucleon scale. We have demonstrated that the momentum dependence can effectively stiffen nuclear matter while the usual incompressibility parameter remains relatively low. The conditions for equilibrium with quark phase described by a bag-model equation of at ate have been investigated. We found that the momentum dependence of the badronic mean field acts to decrease the temperature of the phase transition at a given (nonzero) baryon density.

Semiclassical dynamical simulations of nucleas collisions frequently incorporate another momentum-dependent interaction, the so-called Pauli potential. These calculations proceed through an essentially classical description, where the only quantum-mechanical effect taken into account is the identity of the nucleons. The exclusion principle is simulated by the Pauli potential, designed to keep the nucleons apart in classical phase space. With a new graduate studen,, Mr. J.J. Neumann, we started to look into questions related to the Pauli potential (item 15). It is important to realize that with velocity/momentum.dependent potentials the simple relation between canonical momenta and velocities is no longer valid: e.g. nucleons in a ground-stLie nucleus have finite momenta, but zero velocities. This leads to problems with thermodynarnical quantities that are related to the kinematic (not the canonical) momenta. One hopes to cure these difficulties by treating the Pauli potential in the Lagrangian formalism. This is the approach taken in our work with Mr. Neumann. We have studied the two-body problem and demonstrated that the Fermi gas can be simulated this way; next we will move on to nuclear matter, always carefully distinguishing between canonical and kinematical quantities.

\section{Quark-gluon plasma}

A major development of this research program at Kent State University is the new focus on quark-gluon plasm (QGP) is sues. Item $4,7,8,9,10,12,13$ and 14 belong in this category. As expected and outlined in my July 1991 progrent report, my new postdoctoral associate, Dr. D. Seibert is playing a key role in this initiative. Some of the above items represent independent work by Dr. Seibert, others reflect our collaboration, which started in a very promising manner and is producing its first results. 
Items $7,4,9,12,13$ and 14 can be considered as part of a systematic program to address the diagnostics of the QGP. Thu initial (and schematic) discussion was given in a Phys. Rev. Letter by Dr. Seibert (item 7), where he showed that the transverse mass distribution of lepton pairi from tho mesons is highly sensitive to the QCD transition temperature. This was followed up by including photons as diagnostic tools (items 9 and 12) and by considering more realistic collision scenarios and different assumption sbout the QGP transition (items 4 and 14). Assuming that a transition takes place in the 150-200 MeV temperature range and neglecting transverse expansion, we show that the transverse energy distribution of lepton pairs under the rho peak provides a good 'thermometer' for the transition temperature. Photons can also be used in certain scenarios. Neglecting transverse expansion and assuming thermal and chemical equilibrium throughout the collision are the most restrictive approximations in our work carried out to date. We plan to relax these as future steps of our program.

In addition, Dr. Seibert continues to collaborate with several researchers; the results of one such collaboration are reported in item 10, which proposes a new technique for measuring the ratio of the source transverse velocity to the particle emission velocity, using split-bin correlation functions. This new techrique can be used in the absence of purticle identification, unlike previous techniques.

\section{Modeling of subnucleonic degrees of freedom}

Items 6 and 16 represent steps in our ongoing effort with Dr. P. Tandy, the other (NSF supported) nuclear theorist at KSU, in the theoretical modeling of nuclear matter, psying due attention to the underlying degrees of freedom. Although not immediately practically useful, these calculations are expected to be relevant to the experimental situation (in particular as encountered at CEBAF) at a later stage of their development.

We investigate nuclear matter using a relativistic Hartree approximation to a nonlocal sigma-omega model containing short distance vertex form factors to simulate the underlying QCD substructure, At the Hartree level only the nucleon momentum dependence of the distributed vertices enters, and the resulting finite nonlocal field theory model is solved with simple gaussian forms for the so-called gidew ays forn factors. To reproduce saturated nuclear 
matter the nonlocal model selects form factor ranges at the nucleon muss scale. The model produces a reduced incompressibility and an incrensed effective mass compared to standard point-coupling quantum hadrodynamics (Walecka model). The ralues obtained here sre much easier to reconcile with experimenta information. 

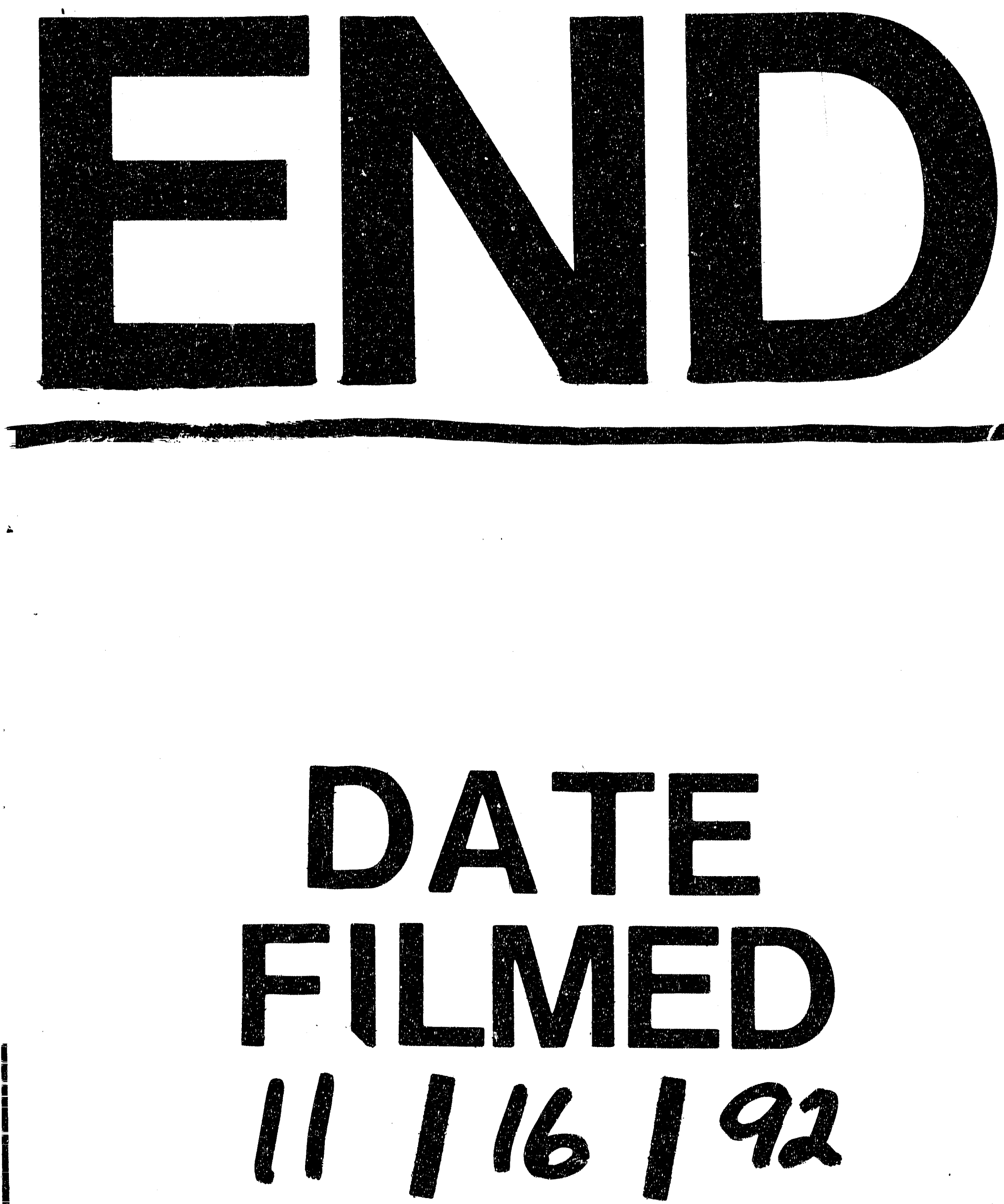
